\title{
Comparison of TFETs and CMOS using optimal design points for power-speed trade-offs
}

\author{
Juan Núñez and María J. Avedillo
}

\begin{abstract}
Tunnel transistors are one of the most attractive steep subthreshold slope devices currently being investigated as a means of overcoming the power density and energy inefficiency limitations of CMOS technology. In this paper, the evaluation and the comparison of the performance of distinct fan-in logic gates, using a set of widely accepted power-speed metrics, are addressed for five projected tunnel transistor (TFET) technologies and four MOSFET and FinFET transistors. The impact of logic depth, switching activity and minimum supply voltage has been also included in our analysis. Provided results suggest that benefits in terms of a certain metric, in which a higher weight is placed on power or delay, are strongly determined by the selected device. Particularly, the suitability of two of the explored TFET technologies to improve CMOS performance for different metrics is pointed out. A circuit level benchmark is evaluated to validate our analysis.
\end{abstract}

Keywords - Tunnel transistors, Steep subthreshold slope, Energy efficiency, Low supply voltage, Optimal design points.

\section{INTRODUCTION}

Tunnel transistors (TFETs) are currently receiving a lot of attention as potential candidates to substitute or complement CMOS devices [1]-[4]. They are one of the most attractive steep subthreshold slope devices. Steep subthreshold slope $(S S)$ enables low voltage operation with acceptable speed leading to power and energy savings. Thus, they are being explored to overcome the power density and energy inefficiency problems exhibited by CMOS due to its $60 \mathrm{mV} /$ decade minimum subthreshold slope [5], [6].

Many works have addressed benchmarking of TFETs at the circuit level with different aims, including the extraction of information useful to guide device design [7], the identification of circuit design challenges or opportunities due to the distinguishing features of these transistors [8]-[10], or the comparison to CMOS to evaluate obtained gains and assessing which applications are candidate for replacing or complementing CMOS with TFETs. In particular, there are a lot of works comparing TFET transistors versus CMOS ones for logic applications [11]-[18].

Manuscript received July 27, 2016, revised October 20, 2016, accepted November 10, 2016. This work has been funded by Ministerio de Economía y Competitividad del Gobierno de España with support from FEDER (Project TEC2013-40670-P).

Juan Núñez and María J. Avedillo are with the Instituto de Microelectrónica de Sevilla, IMSE-CNM (CSIC/Universidad of Sevilla). Av. Américo Vespucio s/n, 41092, Sevilla, Spain. e-mail: \{jnunez/avedillo\}@imse-cnm.csic.es.

Copyright (c) 2016 IEEE. Personal use of this material is permitted. However, permission to use this material for any other other purposes must be obtained from the IEEE by sending a request to pubspermissions@ieee.org.
The latter comparative analyses also follow very different approaches and methodologies. On one hand, some of them rely on analytical expressions using a reduced set of technological parameters such as on current, off current, input capacitance and supply voltage $\left(V_{D D}\right)$. Others uses simulations in order to take into account the great impact of specific features of the TFET transistors including super-linear onset, unidirectional conductance, enhanced Miller Capacitance or dominant gate to drain capacitance on their performance [19], [20].

There are also differences in terms of the circuits considered, from the typical FO4 inverter to system level benchmarks. Finally, there are many distinct criteria on which basis tunnel and CMOS technologies are evaluated. Each allows illustrating some aspects of the differences among both types of transistors. In several cases, realizations of a given circuit implemented with TFETs are compared with their CMOS counterpart at iso-performance or iso-power points. This is interesting for the practical scenery of designing circuits under operating frequency targets or under power budgets. In other works [18], [11], [21], the minimum energy point is used as a figure of merit to summarize the energy advantages. That is, energy optimized designs are compared. However, energy is just one metric of the generalized family of metrics of the form $P^{m} D^{n}$ [22] which represents different trade-offs between power and delay. There is a fundamental relationship between the optimal operating points of a design and the generalized design metrics.

In this paper, a comparative analysis in terms of optimization of a set of widely accepted power-delay design metrics is carried out. Main outstanding key point in our simulation based study is the broad set of technologies which are evaluated: five projected TFET technologies and four CMOS, including MOSFETs and FinFETs, designed for both high performance and low power applications. In addition, switching activity, logic depth and minimum supply voltage are taken into account in our analysis. Using $V_{D D}$ as a design parameter, the average energy per operation $(E)$, energy-delay $(E D P)$ product and power-energy product $(P E P)$ have been obtained and their minimum values evaluated and compared.

The rest of the paper is structured as follows. Section II describes the experiments we have carried out. Results obtained are shown and discussed in Section III. In Section IV, 8-bit adders are evaluated and compared. Finally some conclusions are given in Section V. 


\section{EXPERIMENT DESCRIPTION}

\section{A. Transistors}

Five different tunnel transistor models have been used in this work. All of them are available from the nanoHUB website [23]. Two of them have been derived by Pennsylvania State University and the other three by Notre Dame University. They are briefly described below.

\section{TFET models from Pennsylvania State University [24]}

These are look-up table based Verilog-A models for III-V interband TFETs based on calibrated Synopsys TCAD device simulations. The calibrated TCAD TFET models serve as an approximation of full-band atomistic calculation of TFET band diagram and band-to-band tunneling current to generate the DC characteristics. The gate-source and gate-drain capacitance characteristics obtained from the TCAD smallsignal simulation are validated with measured transient characteristics of TFETs. For $p$-channel transistors drivecurrents identical to those of the $n$-channel are assumed. Its gate-capacitance characteristic is obtained from a TCAD simulation to take into account that density of states of electrons and holes can be quite different in III-V materials. Models with gate lengths of $20 \mathrm{~nm}$ are available for both a double gate InAs Homojunction TFET (PSU Hомо) and a double gate GaSb-InAs Heterojunction TFET (PSU HETE$)$.

\section{TFET models from Notre Dame University [25], [26]}

The current model, based on the Kane-Sze formula for tunneling, is valid in all four operating quadrants of the TFET. It uses a simple analytic model of the gate drain capacitance. Model parameters derived for different TFET structures showed good agreement with atomistic or TCAD device simulations. $p$-channel transistors assume identical drive-on currents and capacitances. Gate length for both transistors is $20 \mathrm{~nm}$. In this work, we use a model for a planar InAs doublegate TFET ( $\mathrm{ND}_{\text {Hомо }}$ ), an $\mathrm{AlGaSb} / \mathrm{InAs}$ double gate TFET $\left(\mathrm{ND}_{\mathrm{HETE}, 1}\right)$ and a GaN/InN single gate TFET $\left(\mathrm{ND}_{\mathrm{HETE}, 2}\right)$.

\section{CMOS transistors}

Four different CMOS transistors have been also evaluated for comparison purposes. All of them are predictive models obtained from the PTM web page [27]. The ones selected were those with channel lengths similar to the available TFETs, namely: 22nm MOSFET devices for both high performance (MOSFET $_{\mathrm{HP}}$, nominal $V_{D D}=0.8 \mathrm{~V}$ ) and low power $\left(\right.$ MOSFET $_{\mathrm{LP}}$, nominal $\left.V_{D D}=0.95 \mathrm{~V}\right)$ applications, and $20 \mathrm{~nm}$ FinFET transistors for high performance FinFET $_{\mathrm{HP}}$, nominal $\left.V_{D D}=0.9 \mathrm{~V}\right)$ and for low stand by power $\left(\right.$ FinFET $_{\mathrm{LP}}$, nominal $\left.V_{D D}=0.9 \mathrm{~V}\right)$.

\section{B. Circuits and measurements}

Fig. 1 shows the circuit used to evaluate and compare logic gates with different fan-in. An inverter, a two-input NAND gate (NAND2) and a three-input NAND (NAND3) gate have been evaluated (in blue). Note that gates under test have been loaded with the parallel connection of four minimum inverters and their inputs were not ideal but generated with chains of inverters.

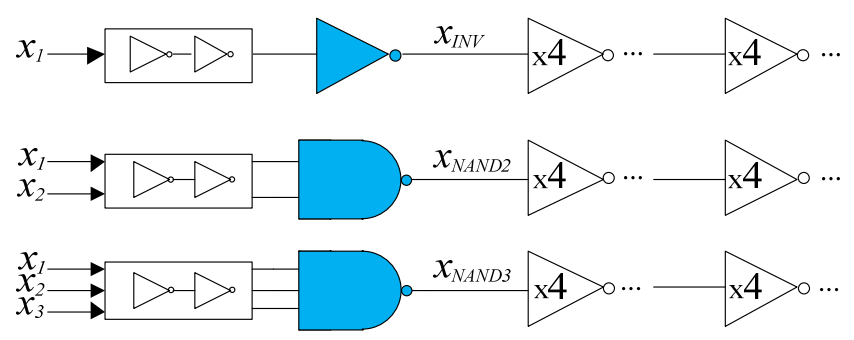

Fig. 1 Circuits used to evaluate the performance of logic gates with different fan in.

\section{Transistor sizing}

In all benchmarking circuits transistors have been sized using minimum gate length. $n$-type transistors width is also the minimum allowable in each case (one finger for the FinFETs). MOSFET $p$-type transistors have been widened (to twice the minimum value) to compensate for mobility differences. Minimum $p$-type TFET transistors have been used since the models already assumed identical drive-on currents.

Applying typical scaling rule, $n$-type transistors have been doubled (multiplied by three) in width in the NAND2 (NAND3) gates to keep similar rise and fall characteristics in all technologies except for FinFETs circuits for which a single finger has been used for all gates.

\section{Measurements}

The benchmarking circuits have been characterized by simulation at different supply voltages in order to take into account the effect of distinctive characteristic of these transistors that impact performance, as mentioned above. Specifically, $V_{D D}$ has been varied from $0.05 \mathrm{~V}$ to $1 \mathrm{~V}$ (with voltage step equal to $0.05 \mathrm{~V}$ ) for CMOS transistors and from $0.05 \mathrm{~V}$ to $0.7 \mathrm{~V}$ for TFET devices. For each circuit and technology, minimum allowable $V_{D D}$ has been determined as the minimum supply voltage at which correct functionality is observed with maximum logic swing degradation of $10 \%$. Worst case high-to-low and low-to-high propagation delays have been measured (at $V_{D D} / 2$ ) and the average of these delays, $\Delta_{F O 4}\left(V_{D D}\right)$, has been used to calculate $f_{M A X}\left(L D, V_{D D}\right)$, the maximum achievable frequency at a given $V_{D D}$ $\left(f_{M A X}\left(L D, V_{D D}\right)=1 /\left(L D \cdot \Delta_{F O 4}\left(V_{D D}\right)\right)\right)$.

Evaluation and comparison in terms of optimization of a set of widely accepted power-delay design metrics, which are members of the generalized family of metrics of the form $P^{m} D^{n}$ [22], have been carried out. Thus, different weights are assigned to power and delay depending on which specification is the most relevant concern.

The average energy per operation $(E)$ has been calculated as $E=P\left(\alpha, f_{M A X}\right) / f_{M A X}$, which corresponds to $m=1$ and $n=1$ in the generalized form of the power-delay metrics. $P\left(\alpha, f_{M A X}\right)$ is the average power consumption for certain values of switching activity $(\alpha)$ and $f_{M A X}$ (and, thus, of $L D$ and $V_{D D}$ ).

We have also evaluated the energy-delay product $(E D P)$ and the power-energy product $(P E P)$ in order to estimate tradeoffs between power and speed performances. EDP and PEP 


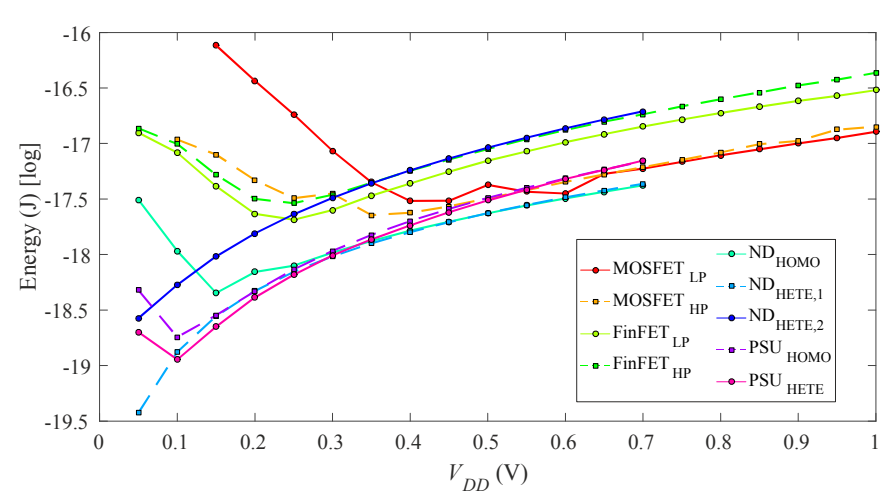

Fig. 2 Energy versus $V_{D D}$ curves for $L D=50$ and $\alpha=0.1$.

have been calculated as follows: $E D P=P\left(\alpha, f_{M A X}\right) / f_{M A X}^{2}(m=1$, $n=2)$ and $P E P=P\left(\alpha, f_{M A X}\right)^{2} / f_{M A X}(m=2, n=1)$.

\section{EVALUATION OF POWER-DELAY METRICS}

In this Section, the performances in terms of energy, EDP and $P E P$ of the circuits shown in Fig. 1 are evaluated and compared. For each figure of merit (described in subsections III.A-III.C), results are firstly discussed in detail for the FO4 inverter and subsequently provided for NAND2 and NAND3 gates.

\section{A. Energy}

Average energy per operation $(E)$ of the $F O 4$ inverter versus $V_{D D}$ curves are shown in Fig. 2 for $L D=50$ and $\alpha=0.1$. The minimum energy for each technological node does not necessarily correspond to the lowest $V_{D D}$. Critical $V_{D D}$ value for minimum energy $\left(V_{D D, O P T}\right)$ is much lower in TFET than in MOSFET/FinFET in agreement with previous works [18], [11], [21], because the on/off current ratio in tunnel technologies is larger (steeper $S S$ ) for low values of $V_{D D}$. In this context, it should be noted that $\mathrm{ND}_{\text {HETE,1 }}$ and $\mathrm{ND}_{\mathrm{HETE}, 2}$ curves have no elbows. It can be also observed that TFET inverters exhibit smaller values of minimum energy than CMOS ones.

The impact of $L D$ and $\alpha$ on the energy performance has been illustrated in Fig. 3. In Fig. 3a, $L D$ has been reduced to 25, without significantly modifying the energy curves but for slight differences in $V_{D D, O P T}$. On the other side, a downward shift of the energy is observed when the switching activity factor is decreased by 10 , as shown in Fig. $3 b$ for $\alpha=0.01$. Note that in Fig. 3a $L D$ was only divided by 2 (doubling frequency for a given $V_{D D}$ ), which explains the more remarkable impact of $\alpha$ variation with respect to the experiment in which $L D$ is varied.
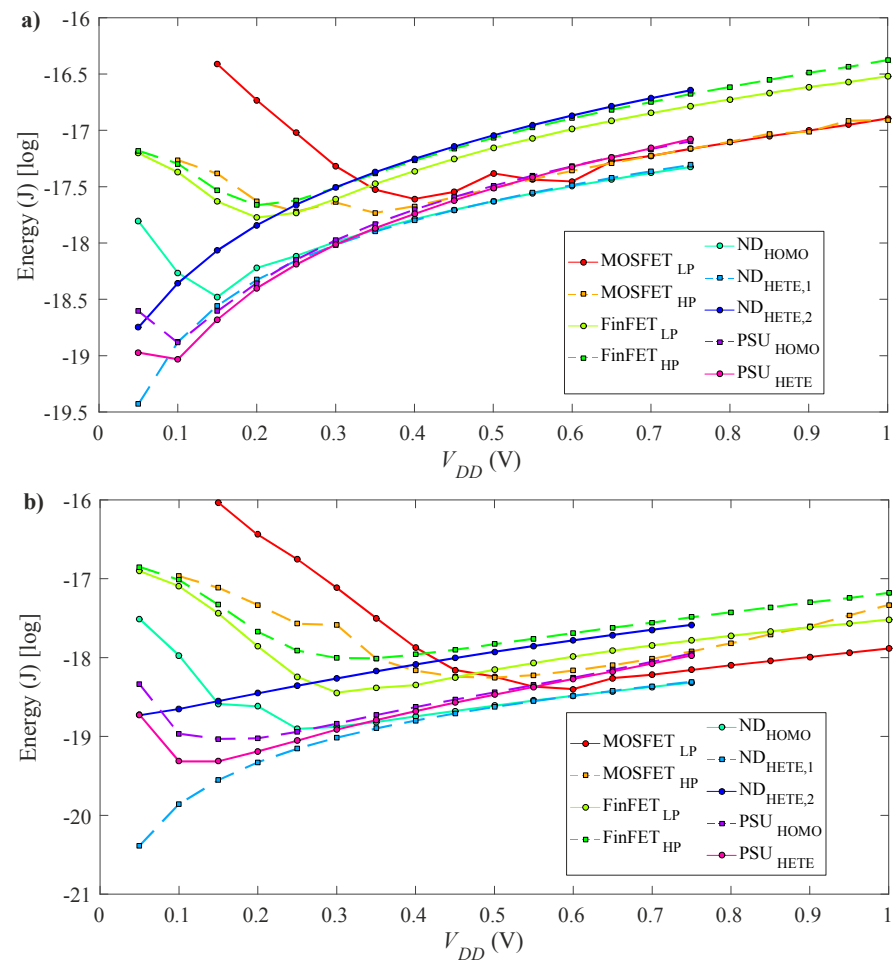

Fig. 3 Impact of $L D$ and $\alpha$ in energy versus $V_{D D}$ curves. (a) $L D=25, \alpha=0.1$. (b) $L D=50, \alpha=0.01$.

To complete the analysis, minimum average energy per operation has been evaluated for six $L D$ and $\alpha$ combinations and each technology. Results are reported in Table I.

Minimum energies among all the technologies are observed in $\mathrm{ND}_{\mathrm{HETE}, 1}$ inverters (marked in red in the Table) for $\alpha=0.01$ and $\alpha=0.1$. However they are obtained for low frequencies: $1.09 \mathrm{MHz}(L D=50)$ and $2.18 \mathrm{MHz}(L D=25)$ and at the minimum $V_{D D}$. For $\alpha=0.5$, PSU $\mathrm{HETE}_{\text {is }}$ the most efficient design. The minimum energy is achieved at $14.07 \mathrm{MHz}$ $(L D=50)$ and $28.14 \mathrm{MHz}(L D=25)$ with $V_{D D}=0.05 \mathrm{~V}$.

Fig. 4 depicts minimum energy versus frequency of the previous experiments for all CMOS technologies and $\mathrm{ND}_{\mathrm{HETE}, 1}$ and $P_{\text {SSETE }}$ devices. For each technology, we have highlighted the area described by the six solutions. As expected, minimum energy at low frequencies is obtained for

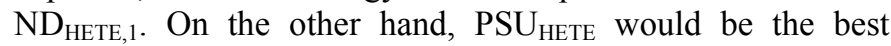
option to achieve high-frequency operation with the lowest energies. Note that MOSFET $_{\mathrm{HP}}$ node exhibits the largest energy values for a wide frequency range and, thus, being not competitive regarding tunnel technologies. Technologies that have not been depicted in Fig. 4 (ND HOMO, $\mathrm{ND}_{\text {HETE, } 2}$ and $\mathrm{PSU}_{\mathrm{HOMO}}$ ) cover the medium energy/frequency range. 
TABLE I. MINIMUM $E, E D P$ AND $P E P$ VALUES FOR $F O 4$ INVERTER

\begin{tabular}{|c|c|c|c|c|c|c|}
\hline \multirow{2}{*}{ Technology } & \multicolumn{6}{|c|}{$(L D, \alpha)$} \\
\hline & $(25,0.01)$ & $(25,0.1)$ & $(25,0.5)$ & $(50,0.01)$ & $(50,0.1)$ & $(50,0.5)$ \\
\hline \multirow{3}{*}{ MOSFET $_{L P}$} & 0.3737 & 2.4606 & 8.8567 & 0.3980 & 3.0362 & 10.3631 \\
\hline & 0.0008 & 0.0075 & 0.0376 & 0.0015 & 0.0151 & 0.0751 \\
\hline & 0.1846 & 0.2971 & 0.3177 & 0.2585 & 0.5891 & 0.6095 \\
\hline \multirow{3}{*}{ MOSFET $_{\mathrm{HP}}$} & 0.4055 & 1.8506 & 2.4745 & 0.5561 & 2.2638 & 4.8003 \\
\hline & 0.0002 & 0.0011 & 0.0049 & 0.0004 & 0.0025 & 0.0102 \\
\hline & 10.0510 & 10.2191 & 10.9841 & 20.0841 & 20.2521 & 21.0061 \\
\hline \multirow{3}{*}{ FinFET $_{L P}$} & 0.2993 & 1.6818 & 4.5602 & 0.3590 & 2.0569 & 6.3455 \\
\hline & 0.0005 & 0.0055 & 0.0273 & 0.0011 & 0.0109 & 0.0546 \\
\hline & 0.2018 & 0.2686 & 0.2825 & 0.2674 & 0.5339 & 0.5476 \\
\hline \multirow{3}{*}{ FinFET $_{\mathrm{HP}}$} & 0.6313 & 2.1621 & 4.5993 & 0.9795 & 2.9028 & 7.7446 \\
\hline & 0.0003 & 0.0023 & 0.0111 & 0.0008 & 0.0048 & 0.0224 \\
\hline & 218.2801 & 191.3001 & 92.9201 & 390.0901 & 412.1501 & 300.5821 \\
\hline \multirow{3}{*}{$\mathrm{ND}_{\text {НОмо }}$} & 0.0994 & 0.3317 & 0.5844 & 0.1246 & 0.45 & 1.1171 \\
\hline & 0.0002 & 0.0022 & 0.0110 & 0.0004 & 0.0044 & 0.02130 \\
\hline & 0.15931 & 0.2712 & 0.2997 & 0.2718 & 0.5356 & 0.5635 \\
\hline \multirow{3}{*}{$\mathrm{ND}_{\text {HETE. } 1}$} & 0.0039 & 0.0373 & 0.1856 & 0.0041 & 0.0375 & 0.1858 \\
\hline & 0.0003 & 0.0028 & 0.0140 & 0.0006 & 0.0056 & 0.0281 \\
\hline & 0.00003 & 0.0030 & 0.0752 & 0.00002 & 0.0015 & $\mathbf{0 . 0 3 7 7}$ \\
\hline \multirow{3}{*}{$\mathrm{ND}_{\text {HETE. } 2}$} & 0.0972 & 0.1785 & 0.5397 & 0.18551 & 0.2668 & 0.6279 \\
\hline & 0.0008 & 0.0032 & 0.0128 & 0.0022 & 0.0079 & 0.0283 \\
\hline & 0.3985 & 1.3425 & 12.2671 & 0.7248 & 1.4988 & 8.3036 \\
\hline \multirow{3}{*}{$\mathrm{PSU}_{\text {номо }}$} & 0.0570 & 0.1299 & 0.3287 & 0.0929 & 0.1801 & 0.4995 \\
\hline & 0.0004 & 0.0030 & 0.0143 & 0.0008 & 0.0061 & 0.0289 \\
\hline & 0.0927 & 0.1239 & 0.2165 & 0.1607 & 0.2283 & 0.3112 \\
\hline \multirow{3}{*}{ PSU $_{\text {HETE }}$} & 0.0278 & 0.0928 & 0.1571 & 0.0483 & 0.1133 & 0.1707 \\
\hline & 0.00003 & 0.0002 & 0.0011 & 0.00008 & 0.0005 & 0.0021 \\
\hline & 0.2532 & 0.3179 & 0.6943 & 0.4381 & 0.5621 & 0.8841 \\
\hline Key & $\begin{array}{c}E[\mathrm{aJ}] \\
E D P[\mathrm{aJ} \cdot \mu \mathrm{s}] \\
P E P\left[\mathrm{aJ}^{2} / \mu \mathrm{s}\right]\end{array}$ & & & & & \\
\hline
\end{tabular}

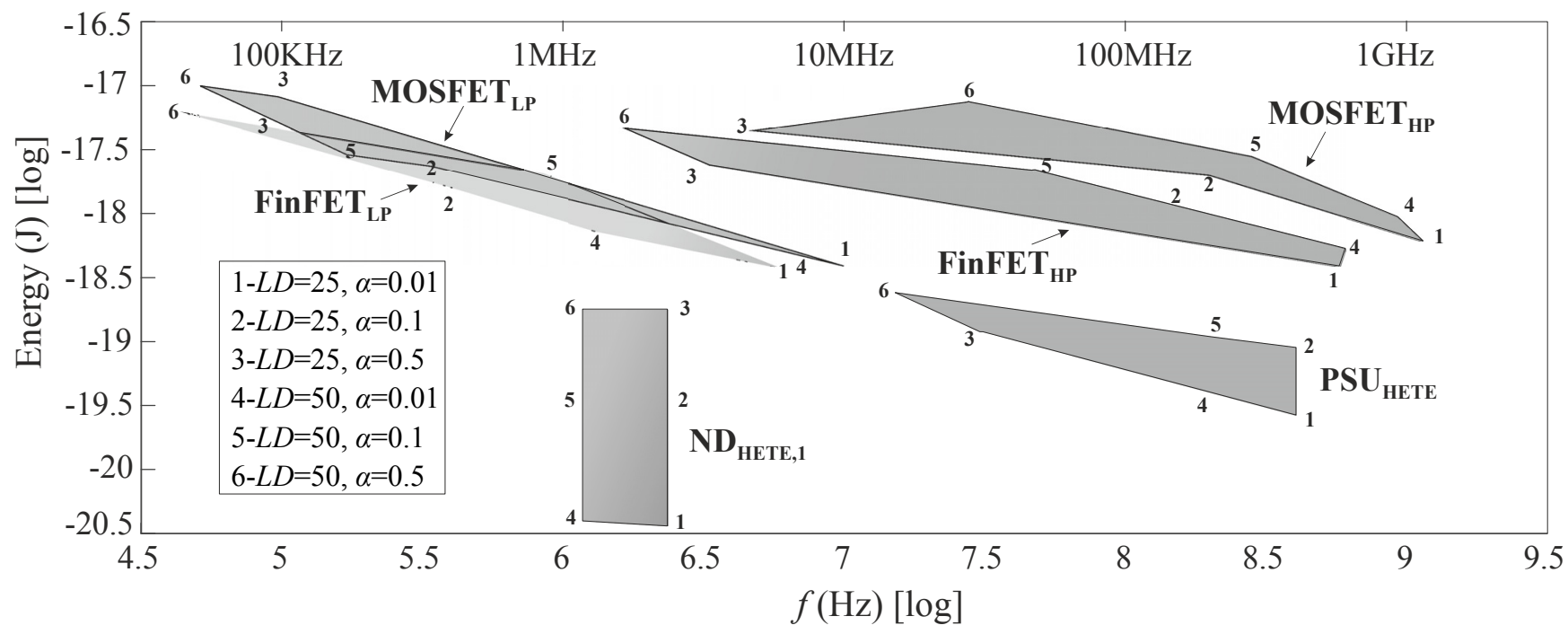

Fig. 4 Minimum energy versus frequency regions for $L D=\{25,50\}$ and $\alpha=\{0.01,0.1,0.5\}$ 

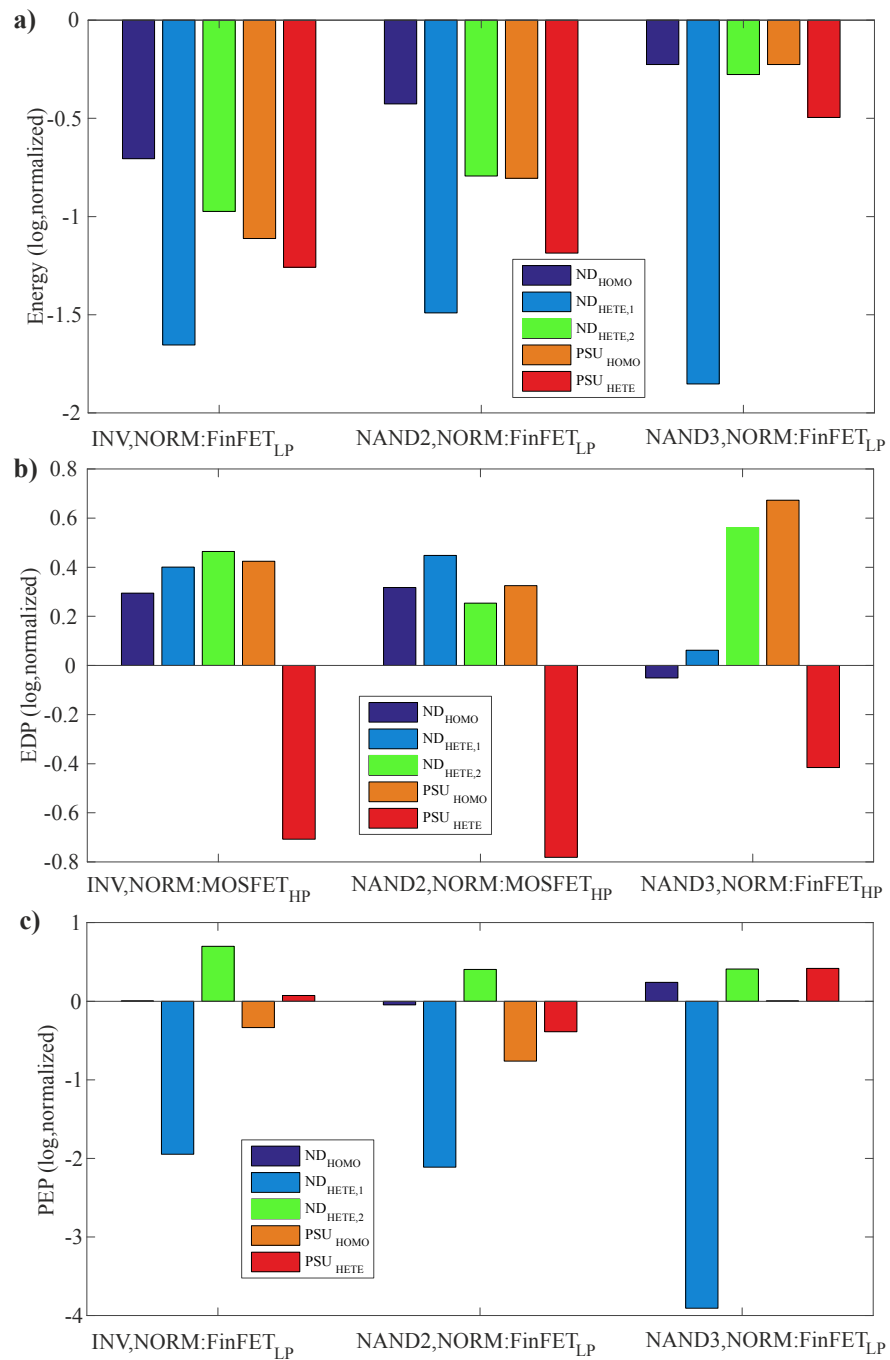

Fig. 5 Bar plots for power-energy metrics for $L D=25$ and $\alpha=0.1$. (a) Minimum energy. (b) Minimum Energy-Delay Product $(E D P)$. (c) Minimum PowerEnergy Product $(P E P)$.

Fig. 5a depicts minimum energy of each tunnel technology, normalized with respect to the best CMOS one, for $L D=25$ and $\alpha=0.1$. Results are provided for the three gates. All values have been represented in logarithmic scale, so that negative values correspond to TFET technologies exhibiting better performance than the best CMOS. Note that, in terms of energy, all TFET inverters are more efficient than the best CMOS technology, FinFET $\mathrm{LP}_{\mathrm{L}}$. As expected, $\mathrm{ND}_{\mathrm{HETE}, 1}$ is the most energy-efficient technology, being 71 (NAND3) to 30

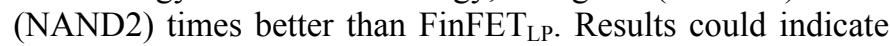
that advantages in terms of the stack factor could be better in

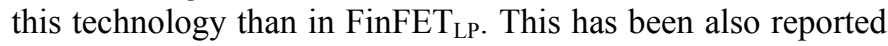
in [7].

It has been also observed that $\mathrm{ND}_{\text {HETE, } 1}$ energy savings are slightly higher for larger values of $L D$. For $L D=50$ it varies from 75 (NAND3) to 33 (NAND2), both compared again to their FinFET $_{\mathrm{LP}}$ counterparts. On the other hand, they are reduced when $\alpha$ increases; i.e. for $\alpha=0.5$ ratios are between 13 (with respect to FinFET $_{\mathrm{LP}}$ inverter) and 55 (FinFET $_{\mathrm{LP}}$ NAND3).

\section{B. Energy-delay product (EDP)}

Results for FO4 inverter minimum EDP in Table I show that PSU $_{\text {HETE }}$ inverter exhibits the best trade-off in terms of $E D P$ for all scenarios (marked in green), in which frequencies between $376 \mathrm{MHz}(L D=25, \alpha=0.01)$ and $1.58 \mathrm{GHz}(L D=25$, $\alpha=0.01)$ are achieved at $V_{D D} \leq 0.20 \mathrm{~V}$.

Results in Fig. $5 \mathrm{~b}(L D=25, \alpha=0.1)$ show that, in terms of $E D P$, PSU $_{\text {HETE }}$ is the most competitive technology for the three

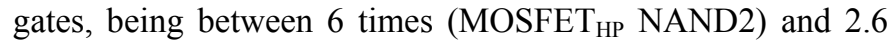
times $\left(\right.$ FinFET $_{\mathrm{HP}}$ NAND3) better than the best MOSFET/FinFET. Note that, as expected, HP CMOS technologies are now the best for the three gates. However, FinFET $_{\text {HP }}$ is the best one only for the NAND3 gate. This result can be explained on the basis of the sizing strategy (described in Section IIII.B), in which wider pull-down transistors are used in MOSFET and TFET gates when the fan-in is increased (stacked transistors) but identical in the FinFET gates. Unlike the inverter and the NAND2, in which normalized EDP results are quite similar, FinFET $\mathrm{HP}_{\mathrm{H}}$ is the most competitive CMOS technology for the NAND3, which explains the apparent degradation of this figure of merit.

None of the other TFET devices in any of the simulated gates are able to improve best CMOS technology but for $\mathrm{ND}_{\text {Hомо in }}$ the NAND3. Finally, it has been verified PSU $\mathrm{HETE}_{\mathrm{E}}$ is the most efficient for the explored $(L D, \alpha)$ design space, where no significant variations on the $E D P$ ratios are observed.

\section{Power-energy product (PEP)}

As shown in Table I for the FO4 inverter, ND HETE, $1_{\text {is the best }}$ technology in terms of minimum $P E P$ (highlighted in blue) for all $(L D, \alpha)$ pairs. In fact, minimum energy designs are also optimum in terms of PEP for $\alpha=\{0.01,0.1\}$. Normalized PEP ratios are depicted in Fig. 5c for the three gates with $L D=25$ and $\alpha=0.1$. Again $\mathrm{ND}_{\mathrm{HETE}, 1}$ is the most efficient technology

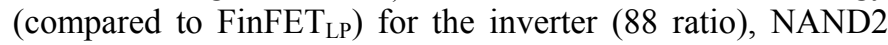
(129) and NAND3 (8040). Again, benefits due to the stack factor compared to FinFET $\mathrm{LP}_{\mathrm{L}}$ are observed, as described above for energy results.

The impact of $L D$ and $\alpha$ variations is very similar to that exhibited for energy results. That is, PEP ratio of $\mathrm{ND}_{\mathrm{HETE}, 1}$ (compared to FinFET $_{\mathrm{LP}}$ ) increases (decreases) when $L D(\alpha)$ does.

\section{Effect of the variation of $V_{D D, M I N}$}

The impact of $V_{D D, M I N}$ on these figures of merits has been analyzed for $\mathrm{ND}_{\mathrm{HETE}, 1}$ (in Fig. 6a) and $\operatorname{PSU}_{\mathrm{HETE}}$ (Fig. 6b) technologies. Similar behavior are observed for both devices: energy savings regarding MOSFET $_{\mathrm{HP}}$ are reduced for larger values of $V_{D D, M I N}$ because, as shown in Fig. 3a, advantages of TFET technologies for low $V_{D D}$ are exploited to a lesser extent. A similar trend is obtained for $P E P$ when $V_{D D, M I N}$ is increased since, for those technologies, also power advantages due to their operation at ultra-low power are weakened. However EDP does not exhibit significant variations because, for both TFET technologies, $V_{D D}$ for minimum $E D P$ is $0.2 \mathrm{~V}$ and, thus, only for the $V_{D D, M I N}=0.25 \mathrm{~V}$ scenario the performance is slightly reduced. 

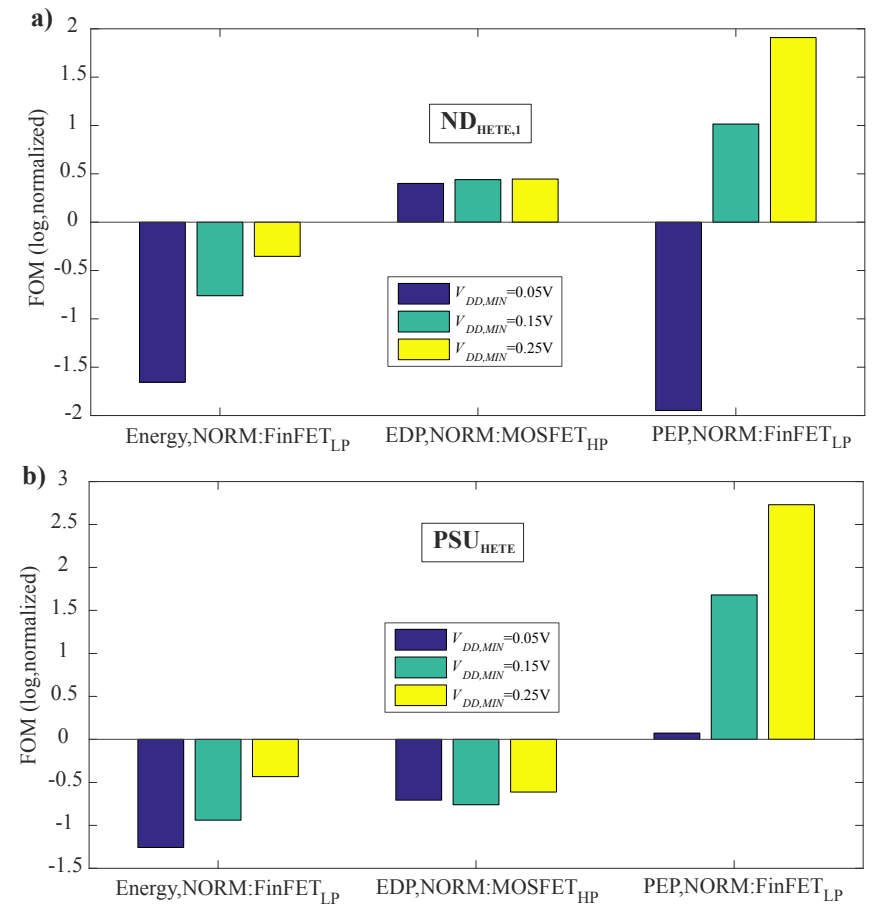

Fig. 6 Impact of $V_{D D, M I N}$ on normalized energy, EDP and PEP for the FO4 inverter designed with $\mathrm{ND}_{\mathrm{HETE}, 1}$ and $\mathrm{PSU}_{\mathrm{HETE}}$ transistors.

\section{CARRY PROPAGATION ADDER RESULTS}

A circuit level benchmark was also included, included in order to take into account some issues, which had not appeared in previous experiments, but which could have an impact on speed or power. These phenomena includes glitches due to the propagation of signals through paths with different delays, leading to extra power, and sustained noise voltage pulses due to capacitive coupling and the asymmetric conduction exhibited by TFET devices, leading to delay degradation [8], [10]. An 8-bit ripple carry adder (RCA) was chosen for this circuit level analysis. An RCA is built by interconnecting fulladders (FA). Fig. 7a shows the logic diagram we used for the FA. Note that it comprises inverters and NAND gates. Average $E, E D P$ and $P E P$ versus $V_{D D}$ curves have been derived for the RCA, similarly to the gate level experiment, and using the ranges of $V_{D D}$ voltages for each technology of the previous experiment. Simulations required to obtain them apply 100 random input combinations.

Fig. 7b shows minimum energy, EDP and PEP for the 8-bit RCA adders implemented with the three heterojunction TFET technologies. The homojunction TFETs are omitted, since they were not shown to be competitive in any of the comparisons carried out at gate level in the previous sections. Results have been normalized with respect to the best CMOS device.

These experiments exhibit significant differences regarding those performed at gate level. Logic paths from the inputs to the outputs are now a combination of different gates (inverter, NAND2 and NAND3) and with different switching activity values. $L D$ is given by the own architecture of the circuit. Note that, since random inputs are applied, large values of $\alpha$ are a)

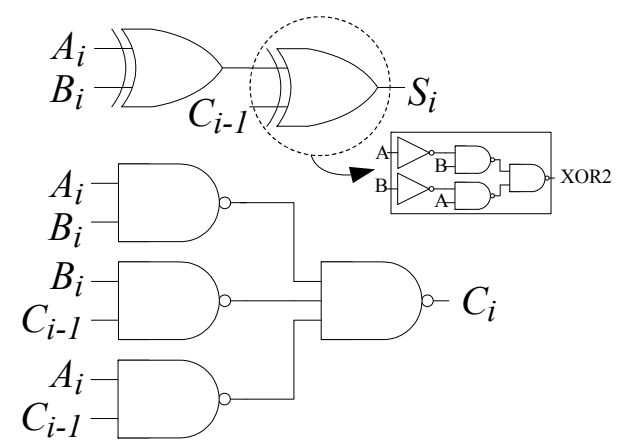

b)

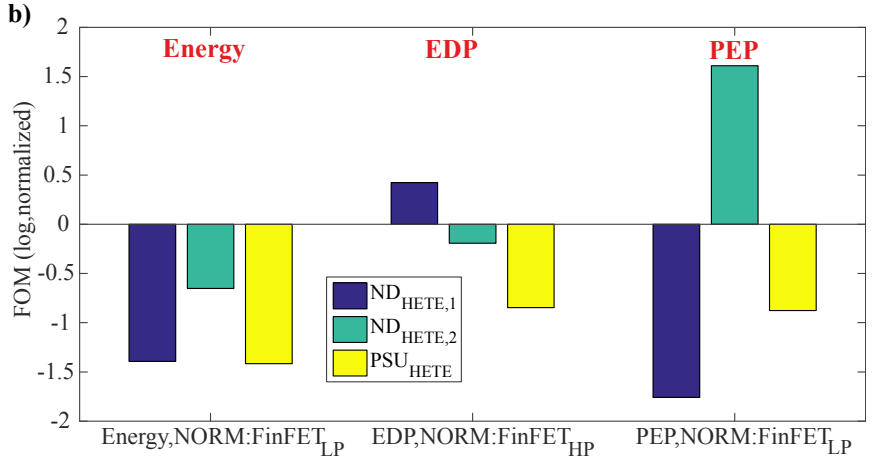

Fig. 7 (a) Logic diagram of one of the full-adders (FA) of an 8-bit ripplecarry adder (RCA). (b) Normalized energy, EDP and PEP of the designed 8bit RCA implemented using the three heterojunction tunnel technologies.

expected. Nonetheless, good agreement with the results obtained at gate level is observed.

Again, all TFET technologies exhibit better energy performance than the CMOS device, being $\mathrm{ND}_{\mathrm{HETE}, 1}$ and PSU $_{\text {HETE }}$ the most efficient technologies. Note that the significant differences between $\mathrm{ND}_{\mathrm{HETE}, 1}$ and $\mathrm{PSU}_{\mathrm{HETE}}$ in Fig. 5a are not observed now. This is due to the distinct switching activity in both experiments. Increasing $\alpha$ has a larger impact in the minimum energy value for $\mathrm{ND}_{\mathrm{HETE}, 1}$ than for PSU $\mathrm{HETE}_{\text {. }}$ This can be observed in Fig. 4 from the shapes of the drawn regions for both technologies. In fact, comparing points 3 (the largest $\alpha$ value represented) for both technologies, it is clear the similarity of the minimum energy values. In terms of $E D P$, PSU $_{\text {HETE }}$ exhibit the best performance, keeping the relative differences with respect to the other TFET devices. Finally, for PEP, $\mathrm{ND}_{\mathrm{HETE}, 1}$ is still the best TFET device, whereas similar trends for the other tunnel devices are observed.

\section{CONCLUSIONS}

In this paper, we evaluate a set of metrics which provides a measure of the performance of distinct circuits implemented using CMOS and TFET technologies. Thus, limitations of a simple estimation of TFET energy savings, achieved by reducing $V_{D D}$ with respect to CMOS technologies, can be overcome. Those metrics include power and delay (frequency) since they are the two most important design specifications. Moreover, we have discussed the impact of switching activity, logic depth or minimum supply voltage on the performance and advantages of TFETs devices.

As in conventional CMOS technologies, a single TFET device is not competitive to optimize a design to achieve a minimum 
value for any metric. Among the five explored TFET technologies in this work, two of them have been identified as candidates to improve CMOS performance. Specifically, $\mathrm{ND}_{\text {HETE, } 1}$ transistors exhibit significant advantages for both energy and PEP with respect to CMOS (even regarding LP devices) since power is weighted equal or higher than frequency. On the other hand, the $\mathrm{PSU}_{\mathrm{HETE}}$ has shown to be greatly advantageous in terms of $E D P$, for which frequency is the primary concern. It is also competitive in terms of energy with respect to $\mathrm{ND}_{\mathrm{HETE}, 1}$ for high switching activity applications. In the circuit level example, only PSU $\mathrm{HETE}_{\mathrm{H}}$ exhibits advantages in the three analyzed figures of merit compared to CMOS technologies.

\section{REFERENCES}

[1] A. Seabaugh and Q. Zhang, "Low-voltage tunnel transistors for beyond CMOS logic," Proceedings of the IEEE, vol. 98, no. 12, Dec. 2010.

[2] A. Seabaugh, "The Tunneling Transistor ", IEEE Spectrum, vol.2, no.4, pp. 55-62, Oct. 2013.

[3] H. Lu and A. Seabaugh, "Tunnel Field-Effect Transistors: State-of-theArt", J. of the Electron Device Society, vol.2, no.4, pp.44-49, Jul. 2014.

[4] U. E. Avci, D.H. Morris and I.A. Young, "Tunnel Field-Effect Transistors: Prospect and Challenges", IEEE Journal of the Electron Dev ice Society, vol. 3, no. 3, pp. 88-95, Jan. 2015.

[5] A.M. Ionescu, H. Riel: "Tunnel field-effect transistors as energy-efficient electronic switches", Nature, no. 479, pp. 329-337, 2011.

[6] H. Chenming; P. Patel; A. Bowonder; J. Kanghoon et al., "Prospect of tunneling green transistor for 0.1V CMOS," Electron Devices Meeting, IEEE International, pp.16.1.1/4, 2010.

[7] D. Esseni, M. Guglielmini, B. Kapidani, T. Rollo, M. Alioto, "Tunnel FETs for Ultra-Low Voltage Digital VLSI Circuits: Part I - DeviceCircuit Interaction and Evaluation at Device Level," IEEE Trans. on VLSI Systems, vol. 22, no. 12, pp. 2488-2498, Dec. 2014.

[8] D.H. Morris, U.E Avci, R. Rios and I.A Young, "Design of low voltage Tunneling-FET logic circuits considering asymmetric conduction characteristics", IEEE Journal on Emerging and Selected Topics in Circuits and Systems, vol. 4, no.4, pp. 380-388, Dec. 2014.

[9] A. Pal, A.B. Sachid, H. Gossner and V.R. Rao, "Insights into the design and optimization of tunnel-FET devices and circuits", IEEE Trans. On Electron Devices, vol. 58, no. 4, pp. 1045-1053, April 2011.

[10] M. J. Avedillo and J. Nuñez, "Improving speed of tunnel FETs logic circuits," IET Electronics Letters, vol.51, no.21, pp.1702-1704, Oct. 2015.

[11] M. Alioto and D. Esseni, "Tunnel FETs for Ultra-Low Voltage Digital VLSI Circuits: Part II-Evaluation at Circuit Level and Design Perspectives", IEEE Trans. On VLSI Systems, vol. 22, no. 12, pp. 24992512, Dec. 2014.

[12] M.J. Avedillo and J. Núñez, "Assessing application areas for tunnel transistor technologies", Proceedings of the XXX Design of Circuits and Integrated Systems Conference (DCIS), pp. 1-6, 2015.

[13] H. Liu, S. Datta, V. Narayanan, "Steep switching tunnel FET: a promise to extend energy efficient roadmap for post-CMOS digital and analog/RF applications", Symp. on Low Power and Design, 2013.

[14] J. Singh; K. Ramakrishnan; S. Mookerjea et.al., "A novel Si-Tunnel FET based SRAM design for ultra low-power 0.3V $V_{D D}$ applications," Design Automation Conf., Asia and South Pacific, pp.181-186,2010.

[15] M. Cotter; L. Huichu; S. Datta; V. Narayanan, "Evaluation of tunnel FET-based flip-flop designs for low power, high performance applications,"14 th Int. Symp. on Quality Elect. Design, pp.430-437, 2013.

[16] K. Swaminathan, M. Seok Kim, N. Chandramoorthy, B. Sedighi, R. Perricone, J. Sampson and V. Narayanan, "Modeling Steep Slope Devices: From Circuits to Architectures", Proceedings Design, Automation and Test in Europe Conference, 2014.

[17] N. Gupta, A. Makosiej, A. Vladimirescu, A. Amara and C. Anghel, ".TTFET bitcell based TFET-CMOS Hybrid SRAM design for Ultra-Low Power Applications", Proceedings Design, Automation and Test in Europe Conference, pp. 361- 366, 2016.

[18] S. Datta, R. Bijesh, H. Liu, D. Mohata and V. Narayanan, "Tunnel Transistors for Low Power Logic", IEEE Compound Semiconductor Integrated Circuit Symposium, pp. 1-4, Oct. 2013.
[19] S. Mookerjea, R. Krishman and S. Datta, "On Enhanced Miller Capacitance Effect in Interband Tunnel Transistors", IEEE Electron Devices Letters, vol. 30, no. 10, pp. 1102-1104, Oct. 2009.

[20] N. Dagtekin and A.M. Ionescu: "Impact of Super-Linear Onset, OffRegion Due to Uni-Directional Conductance and Dominant $\mathrm{C}_{\mathrm{GD}}$ on Performance of TFET-Based Circuits", Journal of the Electron Devices Society, vol. 3, no. 3, pp. 233-239, May 2015.

[21] S. Strangio, P. Palestri, M. Lanuzza, F. Crupi, D. Esseni and L. Selmi, "Assessment of InAs/AlGaSb Tunnel-FET Virtual Technology Platform for Low-Power Digital Circuits", IEEE Trans. On Electron Devices, vol. 63,no.7,pp.2749-2756,July,2016.

[22] D. Sengupta and R. Saleh, "Generalized Power-Delay Metrcis in Deep Submicron CMOS Designs", IEEE Trans. On Computer-Aided Design of Integrated Circuits and Systems, vol.26, no.1, pp.183-189, Jan. 2007.

[23] nanoHUB. https:// nanohub.org.

[24] H. Liu; V. Saripalli; V. Narayanan; S. Datta (2014), "III-V Tunnel FET Model 1.0.0," https://nanohub.org/resources/21012.

[25] H. Lu T. Ytterdal, A. Seabaugh, "Universal TFET model". nanoHUB. doi:10.4231/D3901ZG9H.

[26] H. Lu, D. Esseni, and A. Seabaugh, "Universal analytic model for tunnel FET circuit simulation," Solid-State Electronics, 2015, vol. 108, pp. 110-117, June 2015.

[27] W. Zhao and Y. Cao, "New generation of predictive technology model for sub-45nm design exploration", Proc. $7^{\text {th }}$ Int. Symp. Quality Electronic Design, 2006. http://ptm.asu.edu. 